\title{
A systematic analysis of TCA Escherichia coli mutants reveals suitable genetic backgrounds for enhanced hydrogen and ethanol production using glycerol as main carbon source
}

\author{
Antonio Valle ${ }^{1}$, Gema Cabrera ${ }^{2}$, Howbeer Muhamadali ${ }^{3}$, Drupad K. Trivedi ${ }^{3}$, Nicholas J. W. Ratray ${ }^{3}$, \\ Royston Goodacre ${ }^{3}$, Domingo Cantero ${ }^{2}$ and Jorge Bolivar ${ }^{1}$ \\ ${ }^{1}$ Department of Biomedicine, Biotechnology and Public Health-Biochemistry and Molecular Biology. Campus de Excelencia \\ Internacional Agroalimentario (ceiA3), Institute of Biomolecules (INBIO), University of Cádiz, Puerto Real (Cádiz), Spain \\ ${ }^{2}$ Department of Chemical Engineering and Food Technology. Campus de Excelencia Internacional Agroalimentario (ceiA3), \\ University of Cádiz, Puerto Real (Cádiz), Spain \\ ${ }^{3}$ Manchester Institute of Biotechnology, School of Chemistry, University of Manchester, Manchester, United Kingdom
}

Biodiesel has emerged as an environmentally friendly alternative to fossil fuels; however, the low price of glycerol feed-stocks generated from the biodiesel industry has become a burden to this industry. A feasible alternative is the microbial biotransformation of waste glycerol to hydrogen and ethanol. Escherichia coli, a microorganism commonly used for metabolic engineering, is able to biotransform glycerol into these products. Nevertheless, the wild type strain yields can be improved by rewiring the carbon flux to the desired products by genetic engineering. Due to the importance of the central carbon metabolism in hydrogen and ethanol synthesis, E. coli single null mutant strains for enzymes of the TCA cycle and other related reactions were studied in this work. These strains were grown anaerobically in a glycerol-based medium and the concentrations of ethanol, glycerol, succinate and hydrogen were analysed by HPLC and GC. It was found that the reductive branch is the more relevant pathway for the aim of this work, with malate playing a central role. It was also found that the putative C4-transporter $d c u D$ mutant improved the target product yields. These results will contribute to reveal novel metabolic engineering strategies for improving hydrogen and ethanol production by E. coli.

$\begin{array}{ll}\text { Received } & 05 \text { JAN } 2015 \\ \text { Revised } & \text { 29 MAR } 2015 \\ \text { Accepted } & 04 \text { JUN } 2015 \\ \text { Accepted } & \\ \text { article online } & 09 \text { JUN } 2015\end{array}$

Supporting information available online

Keywords: Bioethanol production - Biohydrogen production · Escherichia coli · Glycerol feed-stocks · Tricarboxylic acid (TCA) cycle

\author{
Correspondence: Dr. Jorge Bolivar, Department of Biomedicine, \\ Biotechnology and Public Health, Campus de Excelencia Internacional \\ Agroalimentario (ceiA3), University of Cádiz, Avda República Saharui s/n, \\ 11510 Puerto Real, Cádiz, Spain \\ E-mails: jorge.bolivar@uca.es \\ Additional correspondence: Dr. Antonio Valle, Department of Biomedi- \\ cine, Biotechnology and Public Health, Campus de Excelencia Interna- \\ cional Agroalimentario (ceiA3), University of Cádiz, Avda República \\ Saharui s/n, 11510 Puerto Real, Cádiz, Spain \\ E-mails: antonio.valle@uca.es
}

Abbreviations: FdhF, formate dehydrogenase-H; FHL, formatehydrogenolyase system; FRQ, fumarate reductase complex; Hyd, hydrogenases; GC/TOF-MS, gas chromatography time of flight-mass spectrometry; MSI, Metabolomics Standards Initiative; QC, quality control; $Y_{\mathrm{E} / \mathrm{X}}$, specific ethanol production; $Y_{\mathrm{G} / \mathrm{X}}$, specific glycerol consumption; $\boldsymbol{Y}_{\mathrm{S} / \mathrm{X}}$, specific succinate efflux

\section{Introduction}

The development of biofuels, which include biodiesel, hydrogen and bioethanol, has become increasingly important due to the current problems associated with the availability and abundance of fossil fuels and environmental pollution [1]. The biodiesel industry has emerged in recent years but the rapid growth of this industry has created excess glycerol, which has resulted in a significant decrease (about 10-fold) in crude glycerol prices and has thus become a burden to the biodiesel industry $[2,3]$. Consequently, glycerol has recently become a cheap source of carbon for biotransformation processes involving microorganisms to give other interesting valuable products, among them ethanol [4-8] and hydrogen [8-10] which are efficient and clean, energy sources [10, 11]. In 
this sense, the production of hydrogen has been recently studied in several microorganisms such as Clostridium, Enterobacter, Lactobacillus and Saccharomyces using glucose, sucrose or xylose as carbon sources [11, 12], although glycerol has also been used in some bacteria. For instance, Enterobacter aerogenes HU-101 has proved to produce $\mathrm{H}_{2}$ with molar yields around $0.8 \mathrm{mmol} \mathrm{H}_{2} / \mathrm{mmol}$ glycerol [5] and $0.88 \mathrm{mmol} \mathrm{H}_{2} / \mathrm{mmol}$ glycerol using Escherichia coli BW25113 wild type strain [13]. Bioethanol can also be obtained by biotransformation of glycerol using several bacteria, including some of those mentioned above [5]. Among the most relevant ethanol producing bacteria are: Enterobacter aerogenes HU-101, Klebsiella sp. HE1 and E. coli whose maximum yields were 0.6-1.0 $\mathrm{mmol}$ ethanol/mmol glycerol [14].

$E$. coli ferments glycerol anaerobically only when complex components, such as yeast extract, tryptone or amino acids, are added to the medium [15]. Whilst the growth rate under these conditions is about 40 times lower than that for aerobic growth in LB medium [7], the culture medium can be optimized to increase the growth rate [16].

In the fermentation of glycerol by $E$. coli two $\mathrm{NAD}^{+}$are reduced, one of them during the conversion of glycerol to DHAP and another one in the conversion of glyceraldehyde 3-P to 1,3-bisphosphoglycerate during the glycolysis (Fig. 1). This fermentation results in a product mixture that predominantly consists of $\mathrm{H}_{2}$ and ethanol but also contains acetate, succinate, and minor amounts of formate [15]. Ethanol and $\mathrm{H}_{2}$ are originated in E. coli after the anaerobic mixed-acid fermentation in which pyruvate, the final product of glycolysis, is fermented to formate, the substrate for $\mathrm{H}_{2}$ synthesis, and acetyl-CoA, which after two reductive steps is transformed to ethanol in which

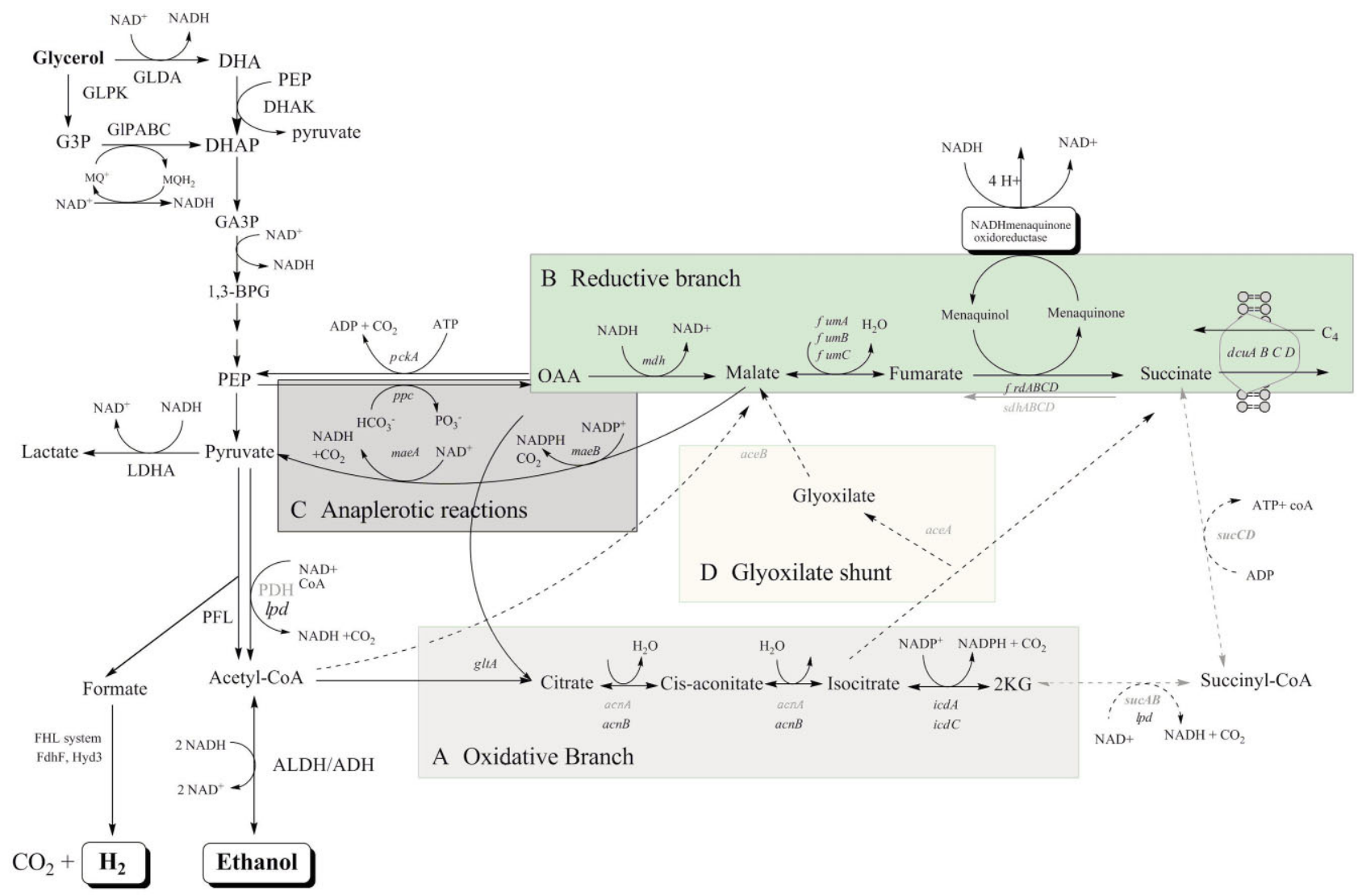

Figure 1. Diagram of TCA during anaerobiosis, which is split into two branches: an oxidative branch (A) and a reductive branch (B) and the associated reactions: anaplerotic reactions (C) and glyoxylate shunt (D). The genes repressed anaerobically are shown in grey and the non-repressed genes in black. The arrows show the reaction directions in accordance with the direction of enzyme catalysis that are theoretically possible in our experimental conditions and the reactions that are theoretically blocked in anaerobic conditions are shown in dash lines. In the oxidative branch (A) the enzymes involved are aconitases ( $A c n A, A c n B$ ), isocitrate dehydrogenases (IcdA, or the putative $I c d C$ ) as well as the associated reactions carried out by 2- $\alpha$-ketoglutarate (2-KG) decarboxylase (SucAB, LpD) and succinyl-CoA synthetase (SucCD) enzymes, which are supposedly inactive. In the reductive branch, the enzymes involved in succinate reduction are malate dehydrogenase (Mdh), fumarase isoenzymes (FumA, FumB, FumC) and fumarate reductase complex (FrdABCD), in which the opposite reaction is involved compared to succinate dehydrogenase (SdhABCD). Succinate can be effluxed by Dcu C4 transporters (DcuA, DcuB, DcuC or the putative DcuD). The anaplerotic reactions (C) involve the phosphoenol pyruvate (PEP) carboxylase (Ppc), which synthesizes oxaloacetate (OAA), and the gluconeogenesis reaction catalysed by PEP carboxykinase (Pck) as well as the malic enzymes (MaeA, MaeB), which synthesize pyruvate from malate. 
two NADH are re-oxidized to balance the NADH/NAD ${ }^{+}$ ratio during the assimilation of glycerol [7]. Therefore, it is crucial to understand the source and use of pyruvate and acetyl-CoA in order to gain a better understanding of $\mathrm{H}_{2}$ and ethanol synthesis in $E$. coli, which may subsequently lead to enhanced production of these two valuable biofuels. Both molecules are tightly connected to the TCA cycle, which under anaerobic fermentative conditions, operates in two oxidative and reductive branch nodes with succinate as the final product of the reductive branch. Succinate can therefore be considered to be a competitive co-product to $\mathrm{H}_{2}$ and ethanol [6] (Fig. 1A and 1B). The reductive branch is also connected to glycolysis by the anaplerotic and pyruvate kinase reactions (Fig. 1C). In aerobiosis E. coli can use acetyl-CoA as a carbon source for biosynthesis by bypassing the two-decarboxylation steps of the TCA cycle through the two reactions of the glyoxylate shunt (Fig. 1D).

Although $E$. coli wild type strains are capable of producing both $\mathrm{H}_{2}$ and ethanol, the yields for both products can be improved by metabolic engineering. For this purpose, several strategies have been developed in the last decade. For instance, it has been described that the blocking of one or several pathways involved in the synthesis of competitive products such as 1,2-propanediol and acetate $[1,13,17-22]$ or the deletion of transporters of precursors, such as the formic transporter FocA and FocB, can improve the production of ethanol and/or $\mathrm{H}_{2}[13,19]$. In this sense, Trchounian and Trchounian have recently described the important role of these formate channels, and how they operate in a pH-dependent manner [23]. Other strategies consisting of the overexpression of enzymes or transcription factors has also been tested. For instance, the overexpression of genes involved in the uptake and conversion of glycerol (GldA) and/or expression of hydrogenase transcriptional factors (FhlA) can help to redirect the carbon flux towards the production of these target products [17-21]. The expression of a desired protein has also been achieved by the elimination of repressor proteins, (i.e. HycA, a repressor of hydrogenase operon) $[1,13,19]$. Another important factor to take into account in the optimization of hydrogen production in $E$. coli is the influence of $\mathrm{pH}$ on the expression and/or the activity of hydrogenase (Hyd-1, 2, 3 and 4) enzymes that are directly involved in the synthesis of $\mathrm{H}_{2}$, as the hydrogenase 3 activity or expression is altered by $\mathrm{pH}$ [24-26]. These strategies have been used to increase the yields of the wild type strain, for instance Tran et al. [13] have described recently how a multiple mutation of seven genes in E. coli increases the molar yield for hydrogen production up to $1.15 \mathrm{mmol} \mathrm{H}_{2} / \mathrm{mmol}_{\text {glycerol consumed and }}$ for ethanol production to $0.92 \mathrm{mmol}$ ethanol/mmol glycerol consumed [13].

In the work described here we studied the role of different enzymes involved in TCA cycle and other related reactions in order to improve our understanding of how the C-flux affects the synthesis of $\mathrm{H}_{2}$ and ethanol when E. coli grows in a glycerol-based medium. With this aim in mind, the production of $\mathrm{H}_{2}$, ethanol and succinate, as well as glycerol consumption, were systematically analysed in single mutant strains obtained from the Keio and Yale collections in which a gene, codifying for one of the aforementioned enzymes, was knocked out. C4 dicarboxylate transporters were also analysed (Fig. 1B) due to they are involved in the uptake and export of C4 TCA cycle metabolites. Several mutants were found with novel phenotypes related to glycerol consumption and hydrogen and ethanol production.

\section{Materials and methods}

\subsection{Bacterial strains and chemicals}

The strain BW25113, a derivative of the $\mathrm{F}^{-}, \mathrm{l}^{-}$, E. coli $\mathrm{K}-12$ strain BD792, was used as the wild type strain (wt) in this work. Isogenic single-gene knock out derivatives [27] were obtained from the National Bioresource Project, Keio Collection (NIG, Japan) and from the Yale University CGSC Stock Center [28], except the double mutant hyaBhybC:.kan which was a gift from T. K. Wood [29]. All of these strains are listed in Supporting information, Table S1.

Kanamycin (Kan) was purchased from Gibco ${ }^{\mathrm{TM}}$ (Invitrogen, UK) and was used for pre-culturing the isogenic knockouts with chromosomal kan resistance

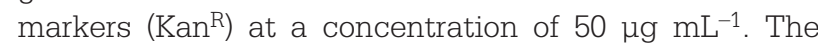
chemicals used for the culture media were as follows: peptone, yeast extracts, agar-agar were obtained from Panreac (PANREAC QUIMICA S.A., Spain) and $\mathrm{KH}_{2} \mathrm{PO}_{4}$, $\mathrm{Na}_{2} \mathrm{HPO}_{4}$ (extra pure), $\mathrm{Na}_{2} \mathrm{SO}_{4}, \mathrm{NaCl}, \mathrm{MgSO}_{4} \cdot 7 \mathrm{H}_{2} \mathrm{O}$ and glycerol (food grade 99\% extra pure) were obtained from Scharlau (Scharlab S.L., Spain).

\subsection{Culture conditions}

E. coli strains listed in Supporting information, Table S1 were initially streaked from glycerol stocks at $-80^{\circ} \mathrm{C}$ on to $\mathrm{LB}$ agar plates containing Kan, and incubated at $37^{\circ} \mathrm{C}$. In this work the mutant strains are also refereed by using $\Delta$ gene. Knock out mutations were confirmed by PCR in each of the mutant strains using external primers (data not shown). In all experiments a single colony was inoculated in $2 \mathrm{~mL}$ of Luria-Bertani (LB) medium and supplemented with Kan, and bacteria were cultured overnight in an orbital incubator shaker at $200 \mathrm{rpm}$ and $37^{\circ} \mathrm{C}$. These cells were used to inoculate (1\% inoculum) $50 \mathrm{~mL}$ of LBGlycerol (LB medium supplemented with glycerol $10 \mathrm{~g} \mathrm{~L}^{-1}$ [109 mM]; $\mathrm{KH}_{2} \mathrm{PO}_{4}, 7.19 \mathrm{~g} \mathrm{~L}^{-1} ; \mathrm{Na}_{2} \mathrm{HPO}_{4}, 1.98 \mathrm{~g} \mathrm{~L}^{-1}$; $\mathrm{Na}_{2} \mathrm{SO}_{4}, 0.0806 \mathrm{~g} \mathrm{~L}^{-1}$, and $\mathrm{MgSO}_{4} \cdot 7 \mathrm{H}_{2} \mathrm{O} 0.031 \mathrm{~g} \mathrm{~L}^{-1}, \mathrm{pH}$ 6.25) previously sparged with argon gas $(99.9 \%)$ for $5 \mathrm{~min}$ to ensure that it was completely deprived of $\mathrm{O}_{2}$. Bacteria 
were grown in $50 \mathrm{~mL}$ Falcon tubes (VWR International Eurolab S.L., Spain) and incubated anaerobically for $\sim 4 \mathrm{~h}$ until an $\mathrm{OD}_{600 \mathrm{~nm}}$ of $\sim 0.6$ was reached. Cultures were centrifuged at $4900 \times g$ for $15 \mathrm{~min}$ at $4^{\circ} \mathrm{C}$ (Sigma 2K15, Laborzentrifugen $\mathrm{GmbH}$, Germany). Inside an anaerobic glove box, previously purged with Ar to diminish the oxygen level to below $1 \%$, the pellet obtained was suspended in approximately $40 \mathrm{~mL}$ of glycerol-based medium [16] $\left(\mathrm{KH}_{2} \mathrm{PO}_{4}, 7.19 \mathrm{~g} \mathrm{~L}^{-1} ; \mathrm{Na}_{2} \mathrm{HPO}_{4}, 1.98 \mathrm{~g} \mathrm{~L}^{-1} ; \mathrm{Na}_{2} \mathrm{SO}_{4}, 0.0806 \mathrm{~g}\right.$ $\mathrm{L}^{-1} ; \mathrm{NaCl}, 0.0152 \mathrm{~g} \mathrm{~L}^{-1} ; \mathrm{MgSO}_{4} \cdot 7 \mathrm{H}_{2} \mathrm{O}, 0.031 \mathrm{~g} \mathrm{~L}^{-1}$; glycerol $10 \mathrm{~g} \mathrm{~L}^{-1}$ [109 mM] and peptone $4.25 \mathrm{~g} \mathrm{~L}^{-1}$, pH 6.25) previously sparged with Ar for $5 \mathrm{~min}$, in order to obtain an $\mathrm{OD}_{600 \mathrm{~nm}}$ of $0.83 \pm 0.025$. The cell suspensions $(4.8 \mathrm{~mL})$ were poured into $12 \mathrm{~mL}$ crimp-top vials and sealed with a butyl rubber septum and aluminium caps. Triplicates of each $E$. coli strain were incubated for 22 and $46 \mathrm{~h}$. The wild type strain was also assayed in the experimental conditions described previously except for the glycerol-based medium $\mathrm{pH}$, which was adjusted at $\mathrm{pH} 7.5$ by using the following salt buffer concentrations: $\mathrm{KH}_{2} \mathrm{PO}_{4}, 1.78 \mathrm{~g} \mathrm{~L}^{-1}$ and $\mathrm{Na}_{2} \mathrm{HPO}_{4}, 7.65 \mathrm{~g} \mathrm{~L}^{-1}$.

\subsection{Metabolomic profiling via gas chromatography- mass spectrometry (GC-MS) analysis}

\subsubsection{Quenching and extraction}

Aliquots of $4.8 \mathrm{~mL}$ of all cell cultures (wt and mutants) taken at 22 and $46 \mathrm{~h}$ time points were immediately quenched in $10 \mathrm{~mL}$ of $60 \%$ aqueous methanol $\left(-48^{\circ} \mathrm{C}\right)$, followed by a centrifugation step at $-9^{\circ} \mathrm{C}$ for $10 \mathrm{~min}$ at $5000 \times \mathrm{g}$ using a Sigma 6K15 centrifuge (Sigma Aldrich, Dorset, UK). The supernatant was removed and samples were snap frozen in liquid nitrogen and stored at $-80^{\circ} \mathrm{C}$. $80 \%$ methanol extraction procedure was employed for metabolite extraction [30]. All extracts were normalized to biomass (3-5 mg) accordingly, followed by combining $100 \mu \mathrm{L}$ aliquots from each of the samples to be used as quality control (OC) samples. Internal standard solution $(100 \mu \mathrm{L}$ of $0.2 \mathrm{mg} \mathrm{mL}^{-1}$ succinic- $d_{4}$ acid, $0.2 \mathrm{mg} \mathrm{mL}{ }^{-1}$ glycine- $d_{5}$, $0.2 \mathrm{mg} \mathrm{mL}^{-1}$ benzoic- $d_{5}$ acid, and $0.2 \mathrm{mg} \mathrm{mL}^{-1}$ lysine- $d_{4}$ ) was added to each of the samples followed by a drying step at $30^{\circ} \mathrm{C}$ for $12 \mathrm{~h}$ using an Eppendorf speed vacuum concentrator (concentrator 5301, Eppendorf, Cambridge, UK). Dried samples were stored at $-80^{\circ} \mathrm{C}$ until further analysis.

\subsubsection{Derivatization and subsequent GC-MS analysis}

Chemical derivatization was carried out by a methoxyamination step followed by trimethylsilylation as described $[31,32]$ in order to give metabolites thermal stability and suitable volatility to enable detection in the gas phase. GC-MS analysis was carried out on a LECO Pegasus III TOF/MS using ChromaTOF v3.0 software following our established protocols and settings as described [33]. This work has been carried out in accordance to the Metabolomics Standards Initiative (MSI) guidelines [34] and has employed a pooled QC procedure, as described in [31], which after deconvolution as described in [32], assisted in statistical evaluation of chromatographic and mass spectral features and the robustness of the data set. All identifications were made from either our own internal mass spectral library, giving an MSI score of 1 and therefore high confidence, or are putative hits from the NIST 8.0 database with an MSI score of 2.

\subsection{Other biochemical measurements}

The volume of gas generated in the headspace was measured by inserting a needle, which was connected to a water column manometer, into the rubber septum. Hydrogen quantification was measured by injecting $100 \mu \mathrm{L}$ aliquots into a Bruker 450-Gas Chromatograph (GC) equipped with a Poraplot Q Plot FS 25X 53 column and a thermal conductivity detector (TCD) (Bruker Daltonik $\mathrm{GmbH}$, Bremen, Germany). The injector and detector were maintained at 250 and $150{ }^{\circ} \mathrm{C}$, respectively and the Ar carrier gas flow rate was maintained at $10 \mathrm{~mL} \mathrm{~min}{ }^{-1}$.

Cell growth was estimated by measuring $\mathrm{OD}_{600 \mathrm{~nm}}$ $(1 \mathrm{OD}=0.31 \mathrm{~g}$ of cell dry weight $(\mathrm{CDW}) / \mathrm{L})$ according to standard procedures [35] on a Spectroquant ${ }^{\circledR}$ Pharo 100 spectrophotometer ( ${ }^{(}$Merck KGaA, Darmstadt, Germany).

Glycerol, ethanol and succinate efflux were obtained from the supernatant of the samples, filtered through $0.22 \mu \mathrm{m}$ nylon filters, and quantified by HPLC as previously described [16].

\subsection{Calculation of parameters and statistical analysis}

Specific hydrogen and ethanol productions $-Y_{\mathrm{H} 2 / \mathrm{X}}$ and $Y_{\mathrm{E} / \mathrm{X}}$ respectively - were calculated with the production values referred to the biomass ( $g$ of CDW) and the specific glycerol consumption $\left(Y_{G / X}\right)$ and the succinate efflux $\left(Y_{\text {Suc/X }}\right)$ were also referred to the biomass.

For each parameter the average $(\mathrm{m})$, standard deviation (S.D.) and the coefficient of variation (CV) were calculated using at least three biological replicates for the mutant strains and at least 75 replicates for the wild type strain. IBM ${ }^{\circledR}$ SPSS $^{\circledR}$ Statistics 20 software was used for statistical analysis and the results for different parameters were considered to be significantly different based on the results of the Non-Parametric Contrast of the Mann-Whitney U Test. The $p$-values used in this Test were 0.01 for

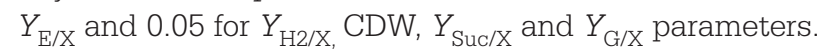
The statistical analysis for the lactic and malic acid normalized areas, was performed by the distribution of continuous variables evaluation with the Shapiro-Wilk's normality test, and the Levene's test for homogeneity of variances was employed to inform the choice of the appropriate statistical test. As the conditions for the application of parametric tests were fulfilled, Student's $t$ test was used for statistically significance of differences of malic and lactic acids peaks between the groups. Molecular and 
functional information for the metabolic pathways used in this work was compiled from Ecocyc [36].

\section{Results}

In order to set up a reference for $\mathrm{H}_{2}$ and ethanol production, the culture conditions were firstly established for the BW25113 strain in order to have a reliable reference to compare several parameters in all the TCA cycle related mutants with those of the wild type strain. The culture medium selected was based on a previous work carried out, in our group by Cofré et al. [7] in which several variables were optimized to obtain an optimal growth. In the present work the specific glycerol consumption $\left(Y_{\mathrm{G} / \mathrm{X}}\right)$ and hydrogen molar yields were studied in the parental strain at five time points, up to $94 \mathrm{~h}$ grown at $\mathrm{pH} 6.25$ and pH 7.5 values. In our experimental condition the glycerol uptake is slightly lower at pH 6.25 than that obtained at pH 7.5 (Supporting information, Fig. S1A), but the hydrogen molar yield was higher at the most acidic $\mathrm{pH}$ (Supporting information, Fig. S1B). As one of the main goals of this study was to study $\mathrm{H}_{2}$ production, the $\mathrm{pH} 6.25$ was chosen. In order to simplify the analysis, 22 and $46 \mathrm{~h}$ post inoculum time points were selected as representative for log and lag phases based on the growth profiles of the wild type strain up to $94 \mathrm{~h}$ (Supporting information, Fig. S1C).

E. coli BW25113 hydrogen and ethanol specific productions $\left(Y_{\mathrm{H} 2 / \mathrm{X}}\right.$ and $\left.Y_{\mathrm{E} / \mathrm{X}}\right)$ and specific glycerol consumption $\left(Y_{\mathrm{G} / \mathrm{X}}\right)$ at these time points were compared with those obtained for all the single mutant strains, available in the KEIO or Yale collections for the genes involved in TCA cycle, as well as for related enzymes that catalyse the anaplerotic and the glyoxylate shunt reactions (Figs. 2, 3 and 4). Succinate efflux was also measured to monitor the carbon efflux and referred to the biomass $\left(Y_{\text {Suc } / X}\right)$. The molar yields for $\mathrm{H}_{2}$, ethanol and succinate efflux ( $\mathrm{mmol}$ product $/ \mathrm{mmol}$ glycerol) were also calculated in those strains whose specific production (mmol product/g CDW) for any of these products were significantly higher than the wild type strain. As controls in our experimental sys-

Figure 2. Plots of parameters in TCA oxidative and related single mutant strains and wild-type (wt) strain: (A) mmol of hydrogen per g CDW $\left(Y_{\mathrm{H} 2 / \mathrm{X}}\right)$, (B) mmol of ethanol per $\mathrm{g}$ of CDW $\left(Y_{\mathrm{E} / \mathrm{X}}\right),(\mathbf{C})$ mmol of glycerol consumed per $\mathrm{g}$ of $\mathrm{CDW}\left(\mathrm{Y}_{\mathrm{C} / \mathrm{X}}\right),(\mathrm{D}) \mathrm{CDW}$ in $\mathrm{g} / \mathrm{L}$ and $(\mathrm{E})$ succinate efflux mmol per $\mathrm{g} C D W\left(Y_{\text {Suc } / \mathrm{X}}\right)$. Bars correspond with the average and standard deviations of every strain in white at $22 \mathrm{~h}$ and in grey at $46 \mathrm{~h}$. Asterisks denote the mutants that are higher, with a statistical significance, than wild-type and < symbols represent the strain values that are lower, with a statistical significance, than wild-type using $p$-value of 0.05 used in the cases of $Y_{\mathrm{H} 2 / \mathrm{X}}(\mathbf{A}), Y_{\mathrm{C} / \mathrm{X}}(\mathbf{C})$ and $\mathrm{CDW}(\mathbf{D})$ and $Y_{\mathrm{Suc} / \mathrm{X}}(\mathrm{E})$ and 0.01 for $\left(Y_{\mathrm{E} / \mathrm{X}}\right)$ (B). Higher asterisks (*) and < symbols denote the significant values at both times. Dashed lines signify the averages of wild-type values at $22 \mathrm{~h}$ (lower line) and $46 \mathrm{~h}$ (upper line) with $n>72$ replicates. For $\operatorname{suc} A$, sucB, sucD and $a c n B$ mutants the values were calculated with $n=6$ replicates and sucC mutants with $n \geq 9$ replicates.] tem, the hydrogen production $\left(Y_{\mathrm{H} 2 / \mathrm{X}}\right)$ in Hydrogenases Hyd-1, 2 and 3 mutants was also analysed (Supporting information, Table S2). In order to facilitate the discussion, these results are arranged according to the pathways described in Fig. 1.

A

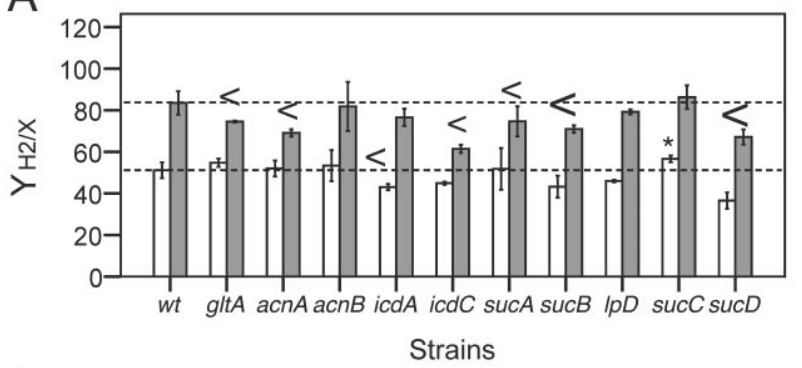

B

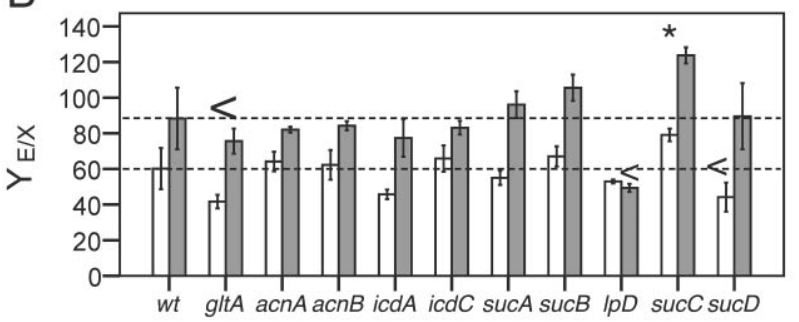

Strains

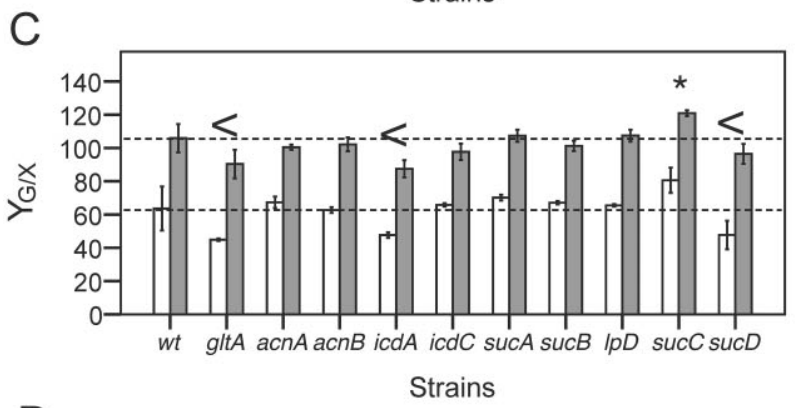

D

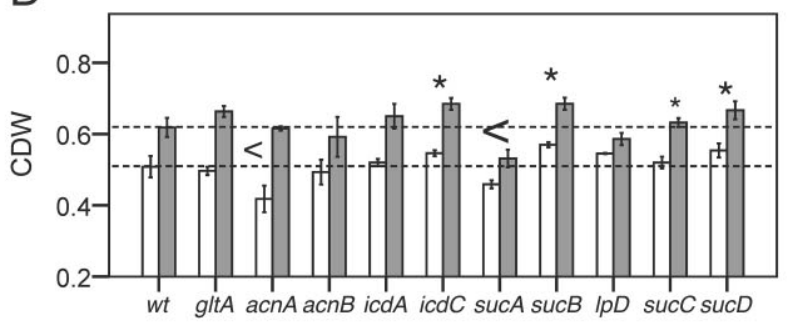

Strains

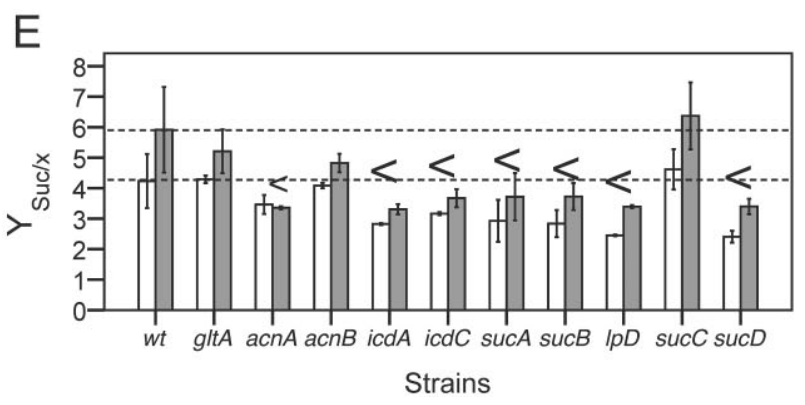




\subsection{Hydrogen and ethanol production, succinate efflux and glycerol consumption in theTCA oxidative branch mutants}

When E. coli grows anaerobically, the condensation of acetyl-CoA and oxaloacetate (OAA) to synthesize citrate is the first reaction in a linear pathway that ends in 2- $\alpha$-ketoglutarate $(2-\mathrm{KG}$ ) because some of the TCA genes (acnA gene and sucABCD operon) are repressed in this environment (Fig. 1A). For most of the oxidative pathway mutants studied in this work ( $g l t A ; \operatorname{acn} A, B ; i c d A, C)$, the specific hydrogen production $\left(Y_{\mathrm{H} 2 / \mathrm{X}}\right)$ was significantly lower than for the wild type strain at $22 \mathrm{~h}$ or $46 \mathrm{~h}$ including the putative isocitrate dehydrogenase mutant $\Delta i c d C$, and only for $\triangle a c n B$ this parameter was similar to that of the wild type at both time points (Fig. 2A). Regarding the specific ethanol production $\left(Y_{\mathrm{E} / \mathrm{X}}\right)$, most of the mutants showed very similar values to the reference wild type, although $\Delta g l t A$ displayed a significantly lower yield at both time points (Fig. 2B). Differences in the specific glycerol consumption $\left(Y_{\mathrm{G} / \mathrm{X}}\right)$ were not observed at both times except for $\Delta g l t A$ and $\Delta i c d A$, for which this parameter was lower than that of the wild type reference (Fig. 2C). In relation to the succinate efflux, all mutants studied in this pathway showed a lower value in comparison to the wild type except for $\Delta g l t A$ and $\Delta a c n B$ (Fig. 2E). When any of these enzyme genes are mutated, the redirection of $\mathrm{C} 4$ provokes a decrease of succinic efflux, although this carbon redirection is not favored towards the synthesis of hydrogen and ethanol.

The synthesis of succinyl-CoA from $2-\alpha$-ketoglutarate by the 2-oxo acid decarboxylase multienzyme complex $(\mathrm{ODH})$ and the subsequent synthesis of succinate by the succinyl-CoA-synthetase (SCS) are theoretically blocked in anaerobiosis [37]; however, the single knock out of the three subunits of the ODH enzyme $(\Delta s u c A, \Delta s u c B$ and $\Delta l p D)$ and the two subunits of the SCS enzyme $(\Delta s u C C$ and $\Delta s u c D$ ) showed different phenotypes when compared to the wild type strain. In this respect, $Y_{\mathrm{H} 2 / \mathrm{X}}$ at $22 \mathrm{~h}$ and $Y_{\mathrm{E} / \mathrm{X}}$ and $Y_{\mathrm{G} / \mathrm{X}}$ at both times were significantly higher in $\Delta$ sucC. By contrast, for the rest of the mutants of both complexes the specific productions were either similar or significantly lower than that of the wild type (Fig. 2A-C). As for the majority of the oxidative branch mutants, $Y_{\text {Suc/X }}$ was significantly lower in these supposedly repressed genes, except in the case of $\Delta s u c C$, which showed no statistically significant differences respect to that of the wild type strain (Fig. 2E).

\subsection{Hydrogen and ethanol production, succinate efflux and glycerol consumption in the TCA reductive branch mutants}

OAA is the starting metabolite of the reductive branch, which after three reductive reactions produces succinate as the final product. Succinate can be exported out of the cells by the C4-dicarboxylate transporters (Fig. 1B), presumably to maintain redox cofactor balances [38].

In the analysis of all mutants involved in this pathway, the malate dehydrogenase mutant $(\Delta m d h)$ was the most markedly affected in terms of all parameters measured at both times under the examined conditions: $Y_{\mathrm{H} 2 / \mathrm{X}}, Y_{\mathrm{G} / \mathrm{X} \text {, }}$ $Y_{\text {Suc/X }}$ were around 0 (Figs. $3 \mathrm{~A}, 3 \mathrm{C}$ and $3 \mathrm{E}$ ) and $Y_{\mathrm{E} / \mathrm{X}}$ was only 0.2 times that of the wild type (Fig. 3B), whilst the cell biomass (CDW) was half the value of the wild type strain (Fig. 3D). There are three fumarase isoenzymes (FumA, FumB and FumC) for the inter-conversion of malate and fumarate in $E$. coli [39]. These enzymes seem to be redundant under the experimental conditions used in this study, although some differences were found between them. In this sense, the $\mathrm{H}_{2}$ specific production was significantly lower to that obtained with the wild type in the case of $\triangle$ fum $C$ at both times while in $\triangle$ fum $A$ was higher at $22 \mathrm{~h}$ but lower at $46 \mathrm{~h}$ (Fig. 3A). $\Delta$ fumA also showed higher $\mathrm{H}_{2}$ molar yield values only at $22 \mathrm{~h}$ (Supporting information, Table S2). However the three fum mutants did not show differences in $Y_{E / X}$ although glycerol consumption was significantly lower for the $\triangle f u m B$ and $\Delta$ fumC strains (Fig. 3B and 3C). Succinate efflux was affected significantly for these three mutants at both times (Fig. 3E).

The last reaction of this pathway is catalysed by FRO, an enzyme composed of four subunits (FrdA, FrdB, FrdC and FrdD). In our analysis, the mutants of the four subunits of FRO showed a tendency towards higher $Y_{\mathrm{H} 2 / \mathrm{X}}$ and $Y_{E / X}$ than the wild type strain (Fig. 3A and 3B). In this sense, the most significant increase in $Y_{\mathrm{H} 2 / \mathrm{X}}, Y_{\mathrm{E} / \mathrm{X}}$ and $Y_{\mathrm{G} / \mathrm{X}}$ was detected in the $\Delta$ frdC strain (Figs. $3 \mathrm{~A}-\mathrm{C}$ ). On the other hand, succinate dehydrogenase encoded by sdh $A B C D$ [40] catalyses the opposite reaction to $F R Q$ and despite the fact that $\Delta s d h A, \Delta s d h B, \Delta s d h C$ and $\Delta s d h D$ are expected to be repressed in anaerobic growth, we found differences between these mutants and the wild type reference except to $\Delta s d h C$ for hydrogen and ethanol production. $\Delta s d h A, \Delta s d h B$ and $\Delta s d h D$ showed a slight but statistically significant lower $Y_{\mathrm{H} 2 / \mathrm{X}}$ at either 22 or $46 \mathrm{~h}$ (Fig. 3A) but significant differences in $Y_{\mathrm{E} / \mathrm{X}}$ were not found except for the $\Delta s d h A$ (Fig. 3B). $Y_{\text {Suc/X }}$ was very similar in all of these mutants to that of the wild type at both times (Fig. 3E).

We also analysed the $\mathrm{C} 4$ membrane transporters in the $\Delta d c u A, \Delta d c u B, \Delta d c u C$ mutants and the putative related but uncharacterized $\Delta d c u D$ (Fig. 3 and 1B). Our results demonstrated, that the levels of the examined metabolites detected in these mutants were similar $\left(d_{u c} B\right)$ or lower $(d c u A$ and $d c u C$ ) than the wild type. However in the case of the previously uncharacterized $\Delta d c u D$ mutant a significant increase in hydrogen ( $20 \%)$ and ethanol production $(\sim 50 \%)$ and glycerol consumption $(\sim 18 \%)$ were detected (Fig. 3). 
A

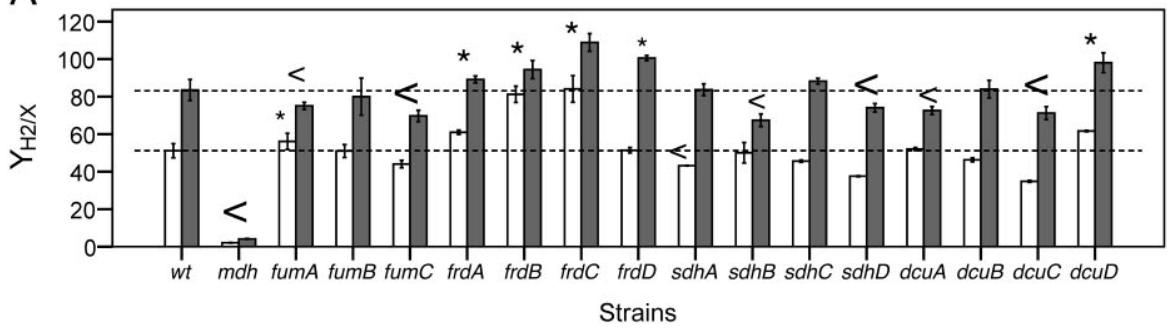

B

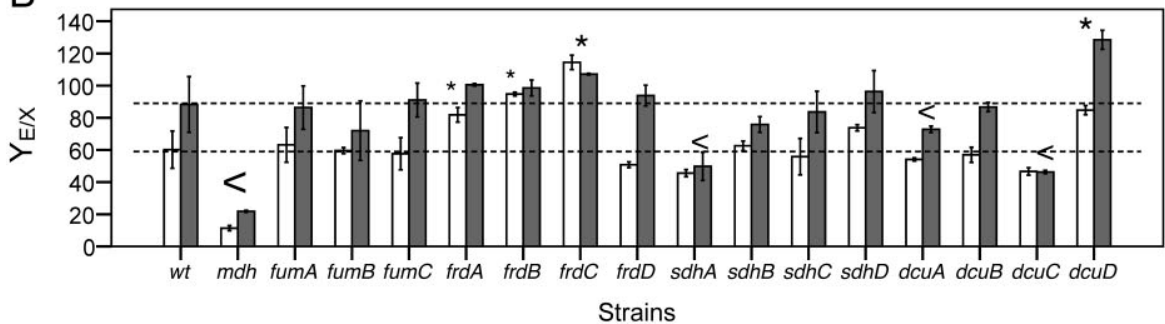

C

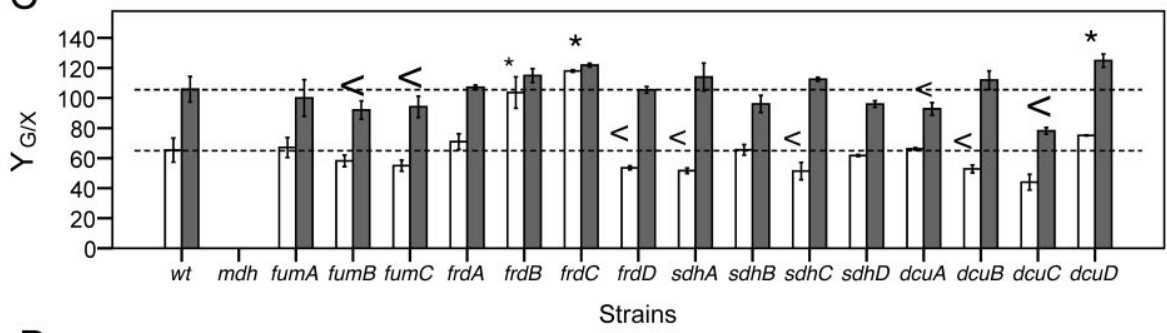

D

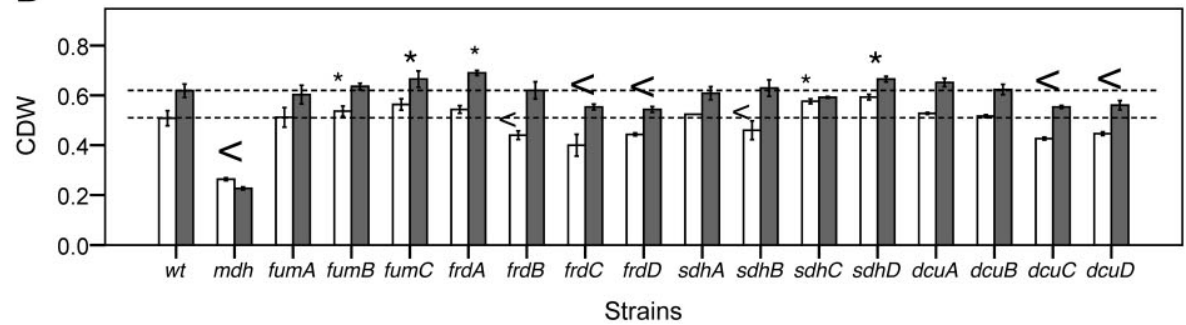

E

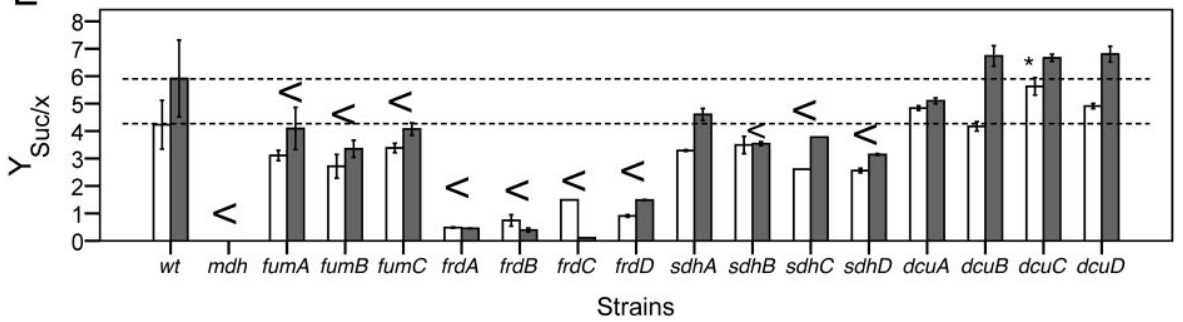

Figure 3. Plots of parameters in TCA reductive branch and $\mathrm{C} 4$ dicarboxylate transporter mutants and wild type strain: (A) mmol of hydrogen per $\mathrm{g}$ CDW $\left(Y_{\mathrm{H} 2 / \mathrm{X}}\right),(\mathbf{B}) \mathrm{mmol}$ of ethanol per $\mathrm{g}$ of $\mathrm{CDW}\left(Y_{E / X}\right),(C) \mathrm{mmol}$ of glycerol consumed per $g$ of CDW $\left(Y_{\mathrm{G} / \mathrm{X}}\right)$, (D) CDW in $\mathrm{g} / \mathrm{L}$ and $(E)$ succinate efflux mmol per $\mathrm{g}$ CDW $\left(Y_{\text {Suc } / X}\right)$. Bars correspond with the average and standard deviations of every strain in white at $22 \mathrm{~h}$ and in grey at $46 \mathrm{~h}$. Asterisks denote the mutants that are higher, with a statistical significance, than wild-type and < symbols represent the strain values that are lower, with a statistical significance, than wild-type using $p$-value of 0.05 used in the cases of $Y_{\mathrm{H} 2 / \mathrm{X}}(\mathbf{A}), Y_{\mathrm{G} / \mathrm{X}}(\mathbf{C})$ and $\mathrm{CDW}(\mathbf{D})$ and $Y_{\text {Suc } / X}(E)$ and 0.01 for $\left(Y_{E / X}\right)(B)$. Higher asterisks (*) and < symbols denote the significant values at both times. Dashed lines signify the averages of wild-type values at $22 \mathrm{~h}$ (lower line) and $46 \mathrm{~h}$ (upper line) with $n>72$ replicates. In the case of fum $A$, fum $B$ and fum $C$ mutants the values were calculated with $n \geq 9$ replicates.

\subsection{Hydrogen and ethanol production, succinate efflux and glycerol consumption in the anaplerotic reactions and glyoxylate shunt mutants}

Both active and supposedly anaerobically repressed anaplerotic genes were studied in this work (Fig. $1 \mathrm{C}$ and 1D). As one would expect, the phenotype in the glyoxylate shunt mutants $(\Delta a c e A$ and $\Delta a c e B)$ is very similar to the wild type strain (Fig. 4A). However, a significant reduction in hydrogen and ethanol production was found for both malic enzyme mutant strains ( $\Delta$ mae $A$ and $\Delta m a e B$ ). For example, $\Delta$ maeA showed a major reduction in $Y_{\mathrm{H} 2 / \mathrm{X}}$ (0.1-fold) and $Y_{\mathrm{E} / \mathrm{X}}(0.2$-fold) yields at both times and in glycerol consumption (Fig. 4A-C). In the case of the $\triangle m a e B$ a reduction by a factor of 0.3 in $Y_{\mathrm{H} 2 / \mathrm{X}}$ and 0.45 in $Y_{\mathrm{E} / \mathrm{X}}$ were measured at $46 \mathrm{~h}$. In the case of the PEP carboxylase $(\Delta p c k)$ and PEP carboxykinase $(\Delta p p c)$ mutants, 
hydrogen production was negatively affected (Fig. 4A) but not so drastically as indicated in $\Delta$ maeA and $\Delta m d h$. The ethanol yield was significantly lower only in $\Delta p c k$ and glycerol consumption significantly decreased in $\Delta p p c$. All of above mutants showed a significantly lower succinate efflux although the most remarkable diminution was measured in $\Delta p c k$ and $\triangle m a e A$ at both times (Fig. $4 \mathrm{~A}$ and 4B).

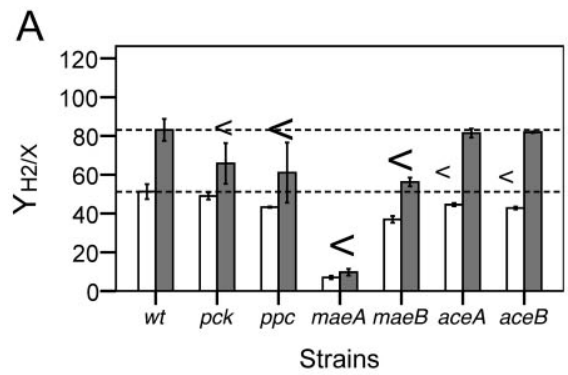

B

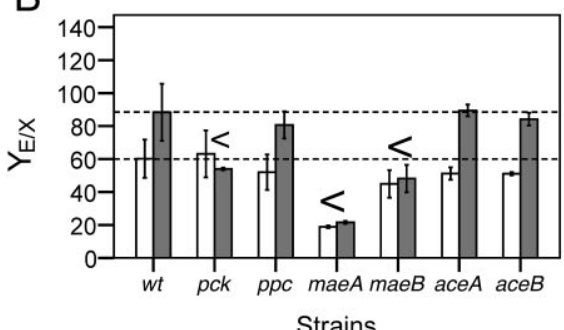

C

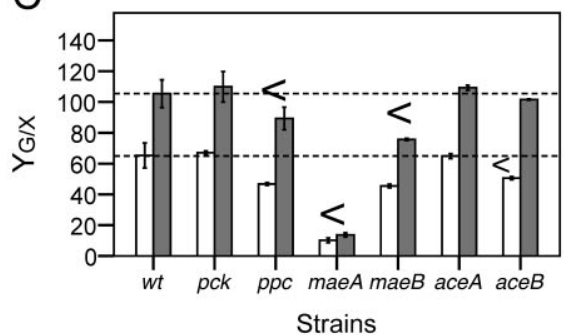

D

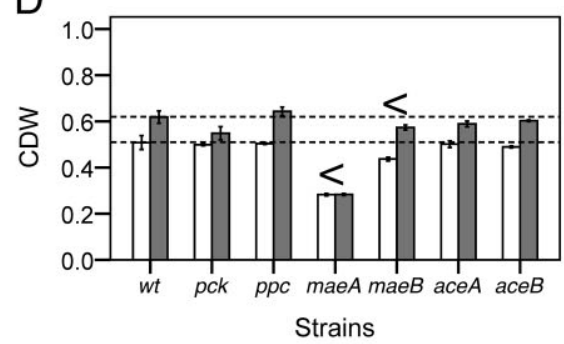

$\mathrm{E}$

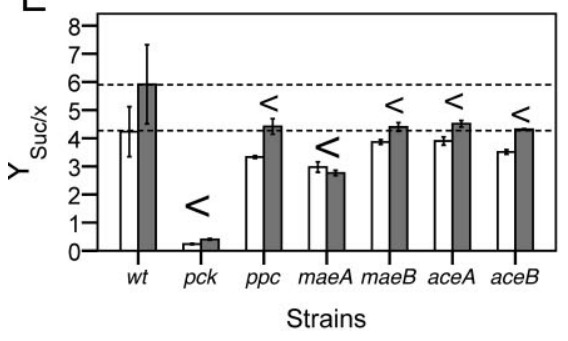

\subsection{Comparative analysis of intracellular malic and lactic acids in the fum $A, f r d A, f r d B$, frdC and $d c u D$ mutants}

The intracellular metabolites of the BW25113 wild type and $\Delta$ fumA, $\Delta$ frdA, $\Delta$ frdB, $\Delta$ frdC and $\Delta d c u D$ were analysed by gas chromatography time of flight mass spectrometry (GC/TOF-MS) as described in Material and method.s. The chromatographic peak areas were normalized to the glycine- $d_{5}$ internal standard (IS). In this analysis 23 metabolites were identified as level 1 of MSI [34] among them malate and lactate, both related to the pathways studied in this work. The peak areas for both metabolites at $22 \mathrm{~h}$ and $46 \mathrm{~h}$ are displayed as box-whisker plots in Fig. 5. The data obtained for malate and lactate were consistent as the QCs, obtained from 42 analytical tests, showed excellent reproducibility and offered high homogeneous data (Fig. 5).

Regarding malate, the integrated peak area was significantly higher in $\triangle$ frdA at $46 \mathrm{~h}$ and in $\Delta d c u D$ at both times in comparison to the wild type strain. Although $\Delta \mathrm{frdB}$ also showed the same tendency to a higher malate intracellular concentration at both times, in this case the differences were not statistically significant. It is remarkable that $\Delta d c u D$ and $\Delta$ frdA displayed malate accumulation during different phases of growth (Fig. 5A). On the other hand, the analysis of intracellular lactate showed that only in the case of the fumA mutant this metabolite was significantly higher than that of the wild type at $46 \mathrm{~h}$. By contrast, the frdB mutant showed lower lactate level at $22 \mathrm{~h}$ compared to the wild type strain and other examined mutants in this study (Fig. 5B).

\section{Discussion}

In this study we aimed to gain an overview of the role of TCA cycle related genes in the production of $\mathrm{H}_{2}$ and ethanol and in the consumption of glycerol in E. Coli during growth on a glycerol-based medium. With this purpose, the culture conditions were firstly established for the BW25113 strain. In this study the pH 6.25 was select-

Figure 4. Plots of parameters in anaplerotic reactions and glyoxylate shunt single mutants and wild type strain: (A) mmol of hydrogen per $\mathrm{g}$ $\mathrm{CDW}\left(Y_{\mathrm{H} 2 / \mathrm{X}}\right)$, (B) mmol of ethanol per g of CDW $\left(Y_{\mathrm{E} / \mathrm{X}}\right),(\mathbf{C}) \mathrm{mmol}$ of glycerol consumed per $g$ of $C D W\left(Y_{C / X}\right),(D) C D W$ in $g / L$ and $(E)$ succinate efflux mmol per g CDW $\left(Y_{\text {Suc } / X}\right)$. Bars correspond with the average and standard deviations of every strain in white at $22 \mathrm{~h}$ and in grey at $46 \mathrm{~h}$. Asterisks denote the mutants that are higher, with a statistical significance, than wild-type and < symbols represent the strain values that are lower, with a statistical significance, than wild-type using $p$-value of 0.05 used in the cases of $Y_{\mathrm{H} 2 / \mathrm{X}}(\mathbf{A}), Y_{\mathrm{G} / \mathrm{X}}(\mathbf{C})$ and $\mathrm{CDW}(\mathbf{D})$ and $Y_{\mathrm{Suc} / \mathrm{X}}(\mathrm{E})$ and 0.01 for $\left(Y_{E / X}\right)$ (B). Higher asterisks (*) and < symbols denote the significant values at both times. Dashed lines signify the averages of wild-type values at $22 \mathrm{~h}$ (lower line) and $46 \mathrm{~h}$ (upper line) with $n>72$ replicates. 

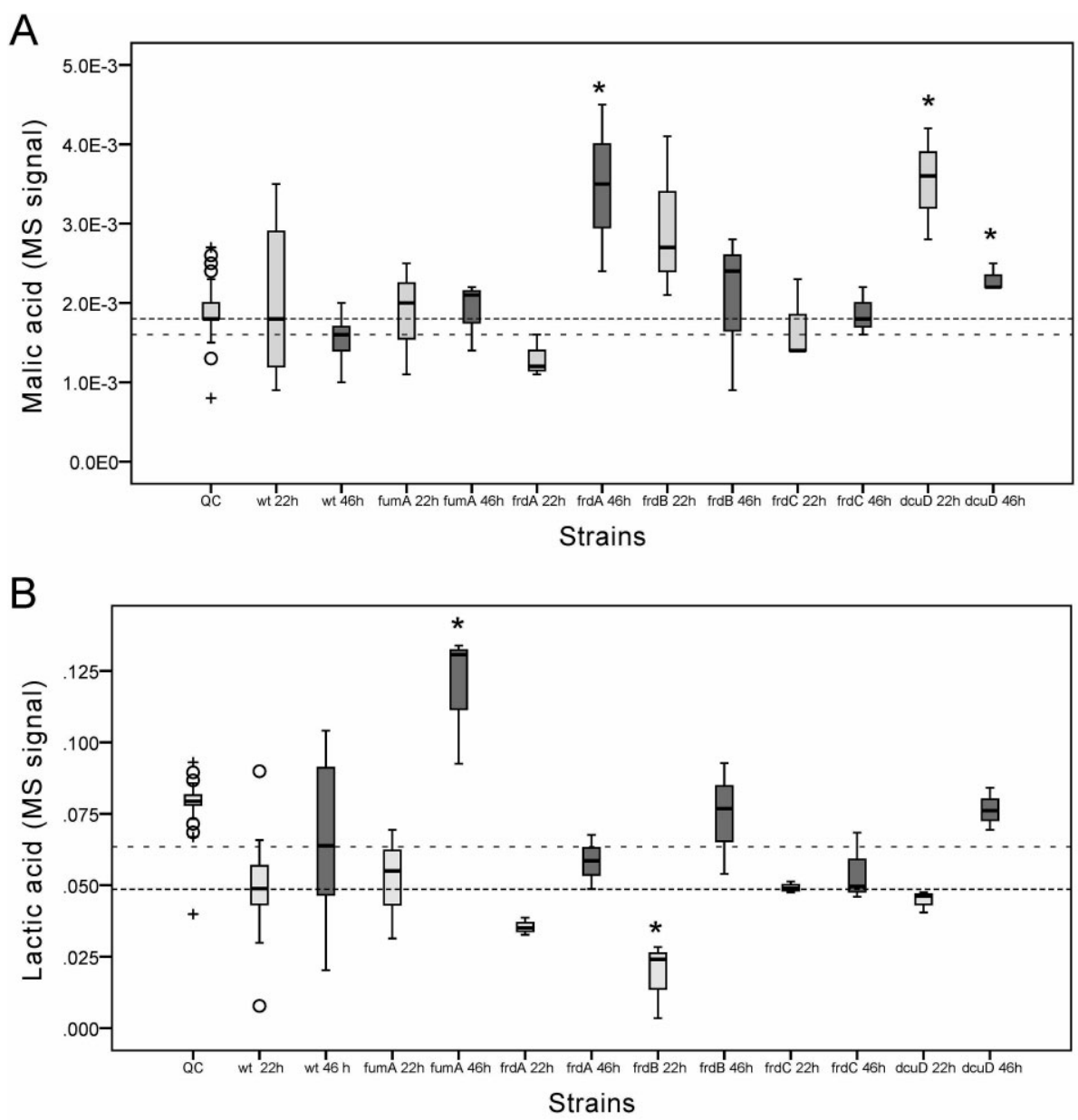

Figure 5. Box plots of malic (A) and lactic acid (B) signal obtained from the GC-MS, normalized by using glycine as internal standard (IS). The clear grey boxes represent the values from samples obtained at $22 \mathrm{~h}$ and the dark grey boxes represent the values obtained at $46 \mathrm{~h}$. The QC boxes are the quality control prepared as described in material and methods. The plots were carried out by using three replicates for the mutant strains at least of 11 replicates, for the wild-type strain (wt). Asterisks denoted the mutants with statistically higher values than wild type. Dashed lines indicate the averages of wild-type values at $22 \mathrm{~h}$ (upper line in pannel $A$ and lower line in pannel B) and $46 \mathrm{~h}$ (lower line in pannel $A$ and upper line in pannel B). ed for the culture medium because acidic $\mathrm{pH}$ had been previously reported as being optimal for Hyd-3 activity $[15,41]$. Although the main goal of the present work was not to deeply describe hydrogen production, several mutants for the hydrogenases 1, 2 and 3 were analysed in order to better understand hydrogen production in this experimental system (Supporting information, Fig. S2). Our results suggests that Hyd-3 is the main gene responsible for hydrogen synthesis in our experimental conditions, since $Y_{\mathrm{H} 2 / \mathrm{X}}$ in $f d h F$ and fhl mutants were very low as have been reported in previous studies [3-6]. By contrast $Y_{\mathrm{H} 2 / \mathrm{X}}$ for the Hyd-1 mutant ( $\triangle$ hyaB) does not seem to play any role in $\mathrm{H}_{2}$ synthesis since $Y_{\mathrm{H} 2 / \mathrm{X}}$ for this mutant was not significantly different with respect to that of the wild type. In the case of Hyd-2 ( $\Delta h y b C)$, the data presented in this work indicate that Hyd-2 is partially involved in the synthesis of hydrogen. The analysis of the double mutant for Hyd-1 and Hyd-2 (Supporting information, Fig. S2) confirmed the null role of Hyd-1 in hydrogen synthesis in our experimental condition. On the other hand, it has also been described that glycerol consumption by E. coli is improved at neutral to slightly alkaline $\mathrm{pH}[4,8,9]$. The data obtained in this work corroborated the different behavior of the wild type strain depending on $\mathrm{pH}$ of the culture medium, which showed a higher $\mathrm{H}_{2}$ molar yield but slightly lower glycerol consumption (Supporting information, Fig. S1A and S1B). During the fermentation process various organic acids are produced that may lower intracellular $\mathrm{pH}$ [13]. As the present work is a comparative analysis, a stable $\mathrm{pH}$ during the experiment was considered a key element in the experimental design.

\subsection{Most of the TCA oxidative branch mutants show a lower hydrogen production}

Some of the genes analysed in this work are supposedly repressed under anaerobic conditions. However, we decided to select blindly all of the available mutants in the Keio or Yale collections for these pathways. It was found that most of the putative repressed mutant strains did not show a significantly different ethanol production when compared to the wild type reference strain. Nevertheless the hydrogen production was significantly lower for most of the oxidative branch mutants. The changes in hydrogen and ethanol production are normally balanced, as for instance, in the case of frd mutants with respect to the 
wild type strain values. This is due to the fact that the precursors of ethanol (acetyl-CoA) and hydrogen (formate) are produced in the same reaction by the pyruvate formatelyase enzyme (PFL) in a 1:1 molar relation. However this is not the result observed in some of the oxidative branch mutants ( $\Delta a c n A, B, \Delta i c d A, C$; Fig. 2). A possible explanation for this divergence could be a partial activation of the pyruvate dehydrogenase complex (PDH) which catalyses the conversion of pyruvate into acetyl-CoA + $\mathrm{CO}_{2}$, but no formate is produced. This activation would lead therefore to an unbalanced acetyl-CoA/formate production. It has been previously described that pyruvate can activate this enzyme by binding to PDH repressor, pyruvate dehydrogenase complex regulator $(\mathrm{PdhR})$. In the $\operatorname{acn} A, B, i c d A, C$ mutants the NADPH production would be impaired due to the inability of isocitrate dehydrogenase (Icd) to catalyse the NADP-dependent oxidative decarboxylation of isocitrate. This situation could be compensated by the NADP-dependent malic enzyme (MaeB) that catalyses the conversion of malate to pyruvate, which could lead to a PDH activation $[42,43]$.

\subsection{The sucC mutant shows a relevant ethanol production}

Surprisingly, $\Delta$ sucA $, \Delta s u c B, \Delta s u c C$ and $\Delta s u c D$ showed differences with respect to the reference strain. Although $\mathrm{ODH}$ and SCS are repressed under anaerobic conditions, a small anaerobic activation of sucA promoter $\left(\mathrm{P}_{\text {suc }}\right)$ has been reported previously [44]. Our data suggest that this could also be the case in our experimental conditions. sucCD and sucAB are mutually essential and low expression of both genes is reported since both sets of genes encode an enzyme that can produce succinyl-CoA, an essential precursor for peptidoglycan biosynthesis [10]. A possible explanation of the higher ethanol production observed in the sucC mutant could be an accumulation of acetyl-CoA and, maybe to a lesser extent, of pyruvate, although this $\mathrm{C}$ rewiring is not related to the reductive branch since succinate efflux is not altered in this mutant. It has been described that suCCD mutant accumulates acetate in aerobic condition [11] and maybe in anaerobic condition this could lead to a higher concentration of acetyl-CoA. In order to elucidate this phenotype, further analysis of intracellular metabolites such as acetate, acetyl-CoA, succinyl-CoA and pyruvate should be needed.

\subsection{Malate plays a central role in the maintaining of $C$ flux and redox balance}

The most notable differences were found in the mutants related to the reductive branch and anaplerotic reactions. The most notable diminution in all the parameters studied in this work was found in $\Delta m a e A$ and $\Delta m d h$, two mutants involved in malate reactions. However the other malic enzyme (MaeB) did not show the same drastic effect as $\Delta m a e A$. This behavior could be due to the different cofactor requirement of the two malic enzymes $\left(\mathrm{NAD}^{+}\right.$ for MaeA and $\mathrm{NADP}^{+}$for $\mathrm{MaeB}$ ) [45]. This could indicate that the $\mathrm{NAD}^{+} / \mathrm{NADH}$ balance, between the $\mathrm{Mdh}$ and MaeA, is essential for the general redox balance of the cell and, in particular, for $\mathrm{H}_{2}$ and ethanol synthesis. Thus, the rewiring of $\mathrm{C} 4$ metabolism to malate could enhance these target product yields.

\subsection{The dcuD mutant is a suitable genetic background to redirect $\mathrm{C}$ flux towards hydrogen and ethanol}

In contrast of these phenotypes we found that the $\mathrm{H}_{2}$ and ethanol specific productions (Fig. 3A and 3B) as well as hydrogen and ethanol molar yields (Supporting information, Table S2) were enhanced when the function of the fumarate reductase (FRQ) is blocked. Similar yields, have been previously described by different authors in $\triangle$ frdA [7], $\Delta$ frdB [46] and $\Delta$ frdC [4, 6, 13]; the $\Delta$ frd $A B$ [47] and the $\triangle$ frdBC [48]. The particularly high values for $Y_{\mathrm{H} 2 / \mathrm{X}}$ in the $\triangle$ frd $C$ could be explained by the attenuation of the negative effect that $\mathrm{H}_{2}$ has on glycerol fermentation in closed vessels previously reported in this mutant by $\mathrm{Hu}$ and Wood [4]. It is noteworthy that the intracellular concentration of malate in $\triangle$ frdA and $\triangle f r d B$ is higher than the wild type, which supports the idea of the central role of this metabolite in the fermentative process (Fig. 5A).

A similar strategy for C4 rewiring could consist of blocking the malate to fumarate conversion, which therefore cannot be transformed to succinate (fum mutants). Whilst variations in the $\mathrm{H}_{2}$ and ethanol yields correlate for most of the mutants, including $\triangle$ fumB and $\Delta$ fum $C$, studied in this work, in the case of FumA a small discrepancy was found between ethanol and $\mathrm{H}_{2}$ yields at this time. The $\Delta$ fum $A$ also showed higher $\mathrm{H}_{2}$ molar yield values only at $22 \mathrm{~h}$ (Supporting information, Table S2). On the other hand, analysis of the intracellular lactate showed that only in this mutant, this metabolite was significantly higher than that of the wild type. The increase of $\mathrm{H}_{2}$ production at $22 \mathrm{~h}$ detected in this mutant could be explained by the changes in the menaquinone-menaquinol oxidoreductase system that could likely occur and may affect to the $\mathrm{NAD}^{+} / \mathrm{NADH}$ balance caused by the increment of synthesize of lactate (Fig. 5B) [49].

We also analysed the C4 membrane transporters dcu family [50] including the putative DcuD [50]. Only the $\Delta d c u D$ showed an increase in hydrogen, and ethanol specific productions and glycerol consumption, which were similar to those of the frd mutants. Remarkably the ethanol molar yield is the highest detected in this work at both times (Supporting information, Table S2). However, these increments do not seem to be due to a redirection of succinate towards the production of the targets products, as the succinate efflux was similar to that of the wild type strain (Fig. 3E). This could indicate that this trans- 
porter normally effluxes an uncharacterized C4 metabolite, possibly malate or a malate precursor, susceptible to be used in pathways related to hydrogen and ethanol synthesis. This hypothesis was corroborated by the GC/TOFMS analysis of the intracellular metabolites since the $d c u D$ mutant accumulates malate at both 22 and $46 \mathrm{~h}$. (Fig. 5A).

From our results we concluded that, out of the two branches in which the TCA cycle is split in anaerobiosis, the reductive branch is the most susceptible to manipulation by genetic modification in order to obtain higher ethanol and $\mathrm{H}_{2}$ yields. $\Delta d c u D$ was identified as suitable genetic background to improve the target products studied in this work. It was also concluded that malate plays an important role in the use of the $\mathrm{C}$ sources in hydrogen and ethanol production as well as in the $\mathrm{NAD}^{+} / \mathrm{NADH}$ balance.

This work was supported by the Consejería de Innovación, Ciencia y Empresa de la Junta de Andalucía through the Project for Excellence P09-TEP-4830 (2009) Co-funded by FEDER Programme 2007-2013 and the PAIDI groups CTS-569 and TEP-105. H.M. and R. G. thank the UK BBSRC for funding H.M.'s PhD. D.K.T. and RG thank the CRUK for funding and we N.J.W.R. and R.G. are also indebted to UK MRC for financial support. Special acknowledgment goes to the NBRP- E. coli at the National Institute of Genetics (Japan) for providing KEIO mutants and the Coli Stock Genetic Center of University of Yale. Special thanks also goes to Thomas K. Wood for the donation of the double mutant $\triangle$ hyaB $\Delta$ hybC.

The authors declare no financial or commercial conflict of interest.

\section{References}

[1] Chen, X., Zhou, L., Tian, K., Kumar, A. et al., Metabolic engineering of Escherichia coli: A sustainable industrial platform for bio-based chemical production. Biotechnol. Adv. 2013, 31, 1200-1223.

[2] Johnson, D. T., Taconi, K. A., The glycerin glut: Options for the value-added conversion of crude glycerol resulting from biodiesel production. Environ. Progress 2007, 26, 338-348.

[3] Yazdani, S. S., Gonzalez, R., Anaerobic fermentation of glycerol: A path to economic viability for the biofuels industry. Curr. Opin. Biotechnol. 2007, 18, 213-219.

[4] Hu, H., Wood, T. K., An evolved Escherichia coli strain for producing hydrogen and ethanol from glycerol. Biochem. Biophys. Res. Commun. 2010, 391, 1033-1038.

[5] Ito, T., Nakashimada, Y., Senba, K., Matsui, T., Nishio, N., Hydrogen and ethanol production from glycerol-containing wastes discharged after biodiesel manufacturing process. J. Biosci. Bioeng. 2005, 100, 260-265

[6] Yazdani, S. S., Gonzalez, R., Engineering Escherichia coli for the efficient conversion of glycerol to ethanol and co-products. Metab. Eng. 2008, 10, 340-351.
[7] Murarka, A., Dharmadi, Y., Yazdani, S. S., Gonzalez, R., Fermentative utilization of glycerol by Escherichia coli and its implications for the production of fuels and chemicals. Appl. Environ. Microbiol. 2008, 74, 1124-1135.

[8] Khanna, S., Goyal, A., Moholkar, V. S., Microbial conversion of glycerol: Present status and future prospects. Crit. Rev. Biotechnol. 2012, 32, 235-262.

[9] Kim, J. H., Choi, K. H., Choi, Y. S., Hydrogen generation from solid $\mathrm{NaBH}_{4}$ with catalytic solution for planar air-breathing proton exchange membrane fuel cells. Int. J. Hydrogen Energ. 2010, 35, 4015-4019.

[10] Panagiotopoulos, I. A., Bakker, R. R., Budde, M. A. W., de Vrije, T. et al., Fermentative hydrogen production from pretreated biomass: A comparative study. Bioresour. Technol. 2009, 100, 6331-6338.

[11] Maeda, T., Sanchez-Torres, V., Wood, T. K., Enhanced hydrogen production from glucose by metabolically engineered Escherichia coli. Appl. Microbiol. Biotechnol. 2007, 77, 879-890.

[12] Goyal, Y., Kumar, M., Gayen, K., Metabolic engineering for enhanced hydrogen production: A review. Can. J. Microbiol. 2013, 59, 59-78.

[13] Tran, K. T., Maeda, T., Wood, T. K., Metabolic engineering of Escherichia coli to enhance hydrogen production from glycerol. Appl. Microbiol. Biotechnol. 2014, 98, 4757-4770.

[14] Adnan, N. A. A., Suhaimi, S. N., Abd-Aziz, S., Hassan, M. A., Phang, L.-Y., Optimization of bioethanol production from glycerol by Escherichia coli SS1. Renewable Energy 2014, 66, 625-633.

[15] Dharmadi, Y., Murarka, A., Gonzalez, R., Anaerobic fermentation of glycerol by Escherichia coli: A new platform for metabolic engineering. Biotechnol. Bioeng. 2006, 94, 821-829.

[16] Cofré, O., Ramírez, M., Gómez, J. M., Cantero, D., Optimization of culture media for ethanol production from glycerol by Escherichia Coli. Biomass Bioenergy 2012, 37, 275-281.

[17] Gonzalez, R., Murarka, A., Dharmadi, Y., Yazdani, S. S., A new model for the anaerobic fermentation of glycerol in enteric bacteria: Trunk and auxiliary pathways in Escherichia coli. Metab. Eng. 2008, 10, 234-245.

[18] Cintolesi, A., Clomburg, J. M., Rigou, V., Zygourakis, K., Gonzalez, R., Quantitative analysis of the fermentative metabolism of glycerol in Escherichia coli. Biotechnol. Bioeng. 2012, 109, 187-198.

[19] Maeda, T., Sanchez-Torres, V., Wood, T. K., Hydrogen production by recombinant Escherichia coli strains. Microb. Biotechnol. 2012, 5, 214-225.

[20] Gonzalez, R., Campbell, P., Wong, M., Production of ethanol from thin stillage by metabolically engineered Escherichia coli. Biotechnol. Lett. 2010, 32, 405-411

[21] Sanchez-Torres, V., Mohd Yusoff, M. Z., Nakano, C., Maeda, T. et al. Influence of Escherichia coli hydrogenases on hydrogen fermentation from glycerol. Int. J. Hydrogen Energ. 2013, 38, 3905-3912.

[22] Trchounian, K., Trchounian, A., Escherichia coli multiple [Ni-Fe]hydrogenases are sensitive to osmotic stress during glycerol fermentation but at different pHs. FEBS Lett. 2013, 587, 3562-3566.

[23] Trchounian, K., Trchounian, A., Different role of focA and focB encoding formate channels for hydrogen production by Escherichia coli during glucose or glycerol fermentation. Int. J. Hydrogen Energ. 2014, 39, 20987-20991.

[24] Trchounian, K., Trchounian, A., Escherichia coli hydrogenase 4 (hyf) and hydrogenase 2 (hyb) contribution in $\mathrm{H}_{2}$ production during mixed carbon (glucose and glycerol) fermentation at pH 7.5 and pH 5.5. Int. J. Hydrogen Energ. 2013, 38, 3921-3929.

[25] Trchounian, K., Trchounian, A., Hydrogenase 2 is most and hydrogenase 1 is less responsible for $\mathrm{H}_{2}$ production by Escherichia coli under glycerol fermentation at neutral and slightly alkaline $\mathrm{pH}$. Int. J. Hydrogen Energ. 2009, 34, 8839-8845.

[26] Trchounian, K., Soboh, B., Sawers, R. G., Trchounian, A., Contribution of hydrogenase 2 to stationary phase $\mathrm{H}_{2}$ production by 
Escherichia coli during fermentation of glycerol. Cell. Biochem. Biophys. 2013, 66, 103-108.

[27] Datsenko, K. A., Wanner, B. L., One-step inactivation of chromosomal genes in Escherichia coli K-12 using PCR products. Proc. Natl. Acad. Sci. U.S.A. 2000, 97, 6640-6645.

[28] Baba, T., Ara, T., Hasegawa, M., Takai, Y. et al., Construction of Escherichia coli K-12 in-frame, single-gene knockout mutants: The Keio collection. Mol. Syst. Biol. 2006, 2, 1-11.

[29] Maeda, T., Sanchez-Torres, V., Wood, T. K., Metabolic engineering to enhance bacterial hydrogen production. Microb. Biotechnol. 2008, 1, 30-39.

[30] Winder, C. L., Dunn, W. B., Schuler, S., Broadhurst, D. et al., Global metabolic profiling of Escherichia coli cultures: An evaluation of methods for quenching and extraction of intracellular metabolites. Anal. Chem. 2008, 80, 2939-2948.

[31] Wedge, D. C., Allwood, J. W., Dunn, W., Vaughan, A. A. et al., Is serum or plasma more appropriate for intersubject comparisons in metabolomic studies? An assessment in patients with small-cell lung cancer. Anal. Chem. 2011, 83, 6689-6697.

[32] Begley, P., Francis-McIntyre, S., Dunn, W. B., Broadhurst, D. I. et al., Development and performance of a gas chromatography-time-offlight mass spectrometry analysis for large-scale nontargeted metabolomic studies of human serum. Anal. Chem. 2009, 81, 70387046 .

[33] Dunn, W. B., Broadhurst, D., Begley, P., Zelena, E. et al., Procedures for large-scale metabolic profiling of serum and plasma using gas chromatography and liquid chromatography coupled to mass spectrometry. Nat. Protoc. 2011, 6, 1060-1083.

[34] Sumner, L. W., Amberg, A., Barrett, D., Beale, M. H. et al., Proposed minimum reporting standards for chemical analysis. Metabolomics 2007, 3, 211-221.

[35] Greenberg, A. E., Clesceri, L. S., Eaton, A. D., Standard Methods for the Examination of Water and Wastewarer. 18th Edition. APHA, Washington 1992, p. 936.

[36] Keseler, I. M., Collado-Vides, J., Gama-Castro, S., Ingraham, J. et al., EcoCyc: A comprehensive database resource for Escherichia coli. Nucleic Acids Res. 2005, 33, D334-337.

[37] Cunningham, L., Guest, J. R., Transcription and transcript processing in the sdhCDAB-sucABCD operon of Escherichia coli. Microbiology 1998, 144, 2113-2123.

[38] Singh, A., Cher Soh, K., Hatzimanikatis, V., Gill, R. T., Manipulating redox and ATP balancing for improved production of succinate in Escherichia coli. Metab. Eng. 2011, 13, 76-81.
[39] Woods, S. A., Schwartzbach, S. D., Guest, J. R., Two biochemically distinct classes of fumarase in Escherichia coli. Biochim. Biophys. Acta 1988, 954, 14-26.

[40] Cecchini, G., Schröder, I., Gunsalus, R. P., Maklashina, E., Succinate dehydrogenase and fumarate reductase from Escherichia coli. Biochim. Biophys. Acta, Gen. Subj. 2002, 15, 140-157.

[41] Bagramyan, K., Mnatsakanyan, N., Poladian, A., Vassilian, A., Trchounian, A., The roles of hydrogenases 3 and 4, and the FOF1ATPase, in $\mathrm{H}_{2}$ production by Escherichia coli at alkaline and acidic pH. FEBS Lett. 2002, 516, 172-178.

[42] Quail, M. A., Guest, J. R., Purification, characterization and mode of action of PdhR, the transcriptional repressor of the pdhR-aceEF-lpd operon of Escherichia coli. Mol. Microbiol. 1995, 15, 519-529.

[43] Ogasawara, H., Ishida, Y., Yamada, K., Yamamoto, K., Ishihama, A., PdhR (pyruvate dehydrogenase complex regulator) controls the respiratory electron transport system in Escherichia coli. J. Bacteriol. 2007, 189, 5534-5541.

[44] Park, S. J., Chao, G., Gunsalus, R. P., Aerobic regulation of the sucABCD genes of Escherichia coli, which encode alpha-ketoglutarate dehydrogenase and succinyl coenzyme A synthetase: Roles of ArcA, Fnr, and the upstream sdhCDAB promoter. J. Bacteriol. 1997, 179, 4138-4142.

[45] Stols, L., Donnelly, M. I., Production of succinic acid through overexpression of $\mathrm{NADH}^{+}$-dependent malic enzyme in an Escherichia coli mutant. Appl. Environ. Microbiol. 1997, 63, 2695-2701.

[46] Agapakis, C. M., Ducat, D. C., Boyle, P. M., Wintermute, E. H. et al., Insulation of a synthetic hydrogen metabolism circuit in bacteria. J. Biol. Eng. 2010, $4,3$.

[47] Seol, E., Manimaran, A., Jang, Y., Kim, S. et al., Sustained hydrogen production from formate using immobilized recombinant Escherichia coli SH5. Int. J. Hydrogen Energ. 2011, 36, 8681-8686.

[48] Yoshida, A., Nishimura, T., Kawaguchi, H., Inui, M., Yukawa, H., Enhanced hydrogen production from glucose using ldh- and frdinactivated Escherichia coli strains. Appl. Microbiol. Biotechnol. 2006, 73, 67-72.

[49] Sawers, R., Clark, D., Fermentative Pyruvate and Acetyl-coenzyme A Metabolism, ASM Press, Washington, DC 2004.

[50] Janausch, I. G., Unden, G., The dcuD (former yhcL) gene product of Escherichia coli as a member of the DcuC family of C4-dicarboxylate carriers: Lack of evident expression. Arch. Microbiol. 1999, 172, 219-226. 


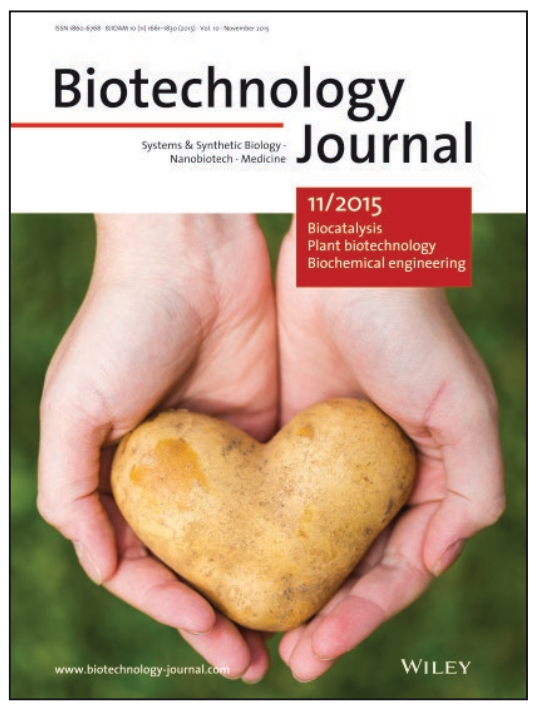

Cover illustration

This regular issue of BT] features articles on the production of biofuels, small molecules and recombinant proteins. The cover is inspired by an article describing increased expression levels of recombinant proteins in potato tubers upon post-harvest light treatment. (C) Lenslife - Fotolia.com

\section{Biotechnology Journal - list of articles published in the November 2015 issue.}

Editorial

Biotechnology Journal brings more than biotechnology

Alois Jungbauer and Sang Yup Lee

http://dx.doi.org/10.1002/biot.201500581

Meeting Report

Plant Science Student Conference (PSSC) 2015 -

Young researchers in green biotechnology

Susann Mönchgesang, Christoph Ruttkies, Hendrik Treutler and Marcus Heisters

http://dx.doi.org/10.1002/biot.201500393

\section{Commentary}

Development of a high affinity Affibody-derived protein against amyloid $\beta$-peptide for future Alzheimer's disease therapy Erwin De Genst and Serge Muyldermans

http://dx.doi.org/10.1002/biot.201500405

\section{Review}

The long-lasting love affair between the budding yeast Saccharomyces cerevisiae and the Epstein-Barr virus María José Lista, Cécile Voisset, Marie-Astrid Contesse, Gaëlle Friocourt, Chrysoula Daskalogianni, Frédéric Bihel, Robin Fåhraeus and Marc Blondel

http://dx.doi.org/10.1002/biot.201500161

Mini-Review

Micro 3D cell culture systems for cellular behavior studies: Culture matrices, devices, substrates, and in-situ sensing methods

Jonghoon Choi, Eun Kyu Lee, Jaebum Choo, Junhan Yuh and Jong Wook Hong

http://dx.doi.org/10.1002/biot.201500092

Review

Outer membrane vesicles as platform vaccine technology Leo van der Pol, Michiel Stork and Peter van der Ley

http://dx.doi.org/10.1002/biot.201400395
Research Article

A truncated and dimeric format of an Affibody library on bacteria enables FACS-mediated isolation of amyloid-beta aggregation inhibitors with subnanomolar affinity Hanna Lindberg, Torleif Härd, John Löfblom and Stefan Ståhl

http://dx.doi.org/10.1002/biot.201500131

Research Article

Enhanced glutathione production by evolutionary engineering of Saccharomyces cerevisiae strains

Anett Patzschke, Matthias G. Steiger, Caterina Holz,

Christine Lang, Diethard Mattanovich and Michael Sauer

http://dx.doi.org/10.1002/biot.201400809

Research Article

A perfusion bioreactor system efficiently generates cell-loaded bone substitute materials for addressing critical size bone defects

Claudia Kleinhans, Ramkumar Ramani Mohan,

Gabriele Vacun, Thomas Schwarz, Barbara Haller,

Yang Sun, Alexander Kahlig, Petra Kluger,

Anna Finne-Wistrand, Heike Walles and Jan Hansmann

http://dx.doi.org/10.1002/biot.201400813

Research Article

Biocatalyzed approach for the surface functionalization of poly(L-lactic acid) films using hydrolytic enzymes Alessandro Pellis, Enrique Herrero Acero, Hansjoerg Weber, Michael Obersriebnig, Rolf Breinbauer, Ewald Srebotnik and Georg M. Guebitz

http://dx.doi.org/10.1002/biot.201500074 
Research Article

A systematic analysis of TCA Escherichia coli mutants reveals suitable genetic backgrounds for enhanced hydrogen and ethanol production using glycerol as main carbon source Antonio Valle, Gema Cabrera, Howbeer Muhamadali, Drupad K. Trivedi, Nicholas J. W. Ratray, Royston Goodacre, Domingo Cantero and Jorge Bolivar

http://dx.doi.org/10.1002/biot.201500005

Research Article

Engineering surface hydrophobicity improves activity

of Bacillus thermocatenulatus lipase 2 enzyme

Ting Tang, Chongli Yuan, Hyun-Tae Hwang, Xuebing Zhao, Doraiswami Ramkrishna, Dehua Liu and Arvind Varma

http://dx.doi.org/10.1002/biot.201500011

Research Article

The potential of random forest and neural networks for biomass and recombinant protein modeling in Escherichia coli fed-batch fermentations

Michael Melcher, Theresa Scharl, Bernhard Spangl,

Markus Luchner, Monika Cserjan, Karl Bayer,

Friedrich Leisch and Gerald Striedner

http://dx.doi.org/10.1002/biot.201400790

Research Article

Determination of antibiotic $\mathrm{EC}_{50}$ using a zero-flow microfluidic chip based growth phenotype assay

Jing Dai, Sang-Jin Suh, Morgan Hamon and Jong Wook Hong http://dx.doi.org/10.1002/biot.201500037
Research Article

A potyvirus vector efficiently targets recombinant proteins to chloroplasts, mitochondria and nuclei in plant cells when expressed at the amino terminus of the polyprotein

Eszter Majer, José-Antonio Navarro and José-Antonio Daròs

http://dx.doi.org/10.1002/biot.201500042

Research Article

Post-harvest light treatment increases expression levels of recombinant proteins in transformed plastids of potato tubers Luis M. Larraya, Alicia Fernández-San Millán, María Ancín, Inmaculada Farran and Jon Veramendi

http://dx.doi.org/10.1002/biot.201500028

Biotech Method

Prediction of reversible lgG 1 aggregation occurring in a size exclusion chromatography column is enabled through a model based approach

Frida Ojala, Anton Sellberg, Thomas Budde Hansen,

Ernst Broberg Hansen and Bernt Nilsson

http://dx.doi.org/10.1002/biot.201500160

Biotech Method

Dual lifetime referencing enables $\mathrm{pH}$-control for oxidoreductions in hydrogel-stabilized biphasic reaction systems

Jens Begemann and Antje C. Spiess

http://dx.doi.org/10.1002/biot.201500198 